\title{
Problemáticas EN LA TRANSICIÓN ECONÓMICA Y SOCIODEMOGRÁFICA DE MÉXICO Y VIETNAM
}

\author{
Lukasz Czarnecki ${ }^{1}$
}

Fecha de recepción: 05 de mayo de 2016. Fecha de aceptación: 26 de agosto de 2016.

\begin{abstract}
RESUMEN
¿Cuáles son los impactos del neoliberalismo en la transición política y sociodemográfica en México y la República Socialista de Vietnam, tomando en cuenta similitudes y diferencias entre ambos países? La dinámica de la transformación del Estado y la sociedad en México y Vietnam se aceleró con el proceso de la implementación de las políticas neoliberales a partir de los años ochenta, modus operandi de la disminución del papel del Estado y su relación con la economía de ambos países. El neoliberalismo también tuvo impacto en la esfera social, al analizar la transición demográfica, creando brechas sociodemográficas entre grupos poblacionales, asimismo en la esfera educativa y laboral, aún más en la población indígena.
\end{abstract}

Palabras clave: políticas neoliberales, crisis del Estado, transición sociodemográfica, educación, población indígena.

Clasificación JEL: A12, A13, J15, J18, Y80.

\section{Challenges in the Economic and Sociodemographic Transition of Mexico and Vietnam}

\begin{abstract}
What are the impacts of neoliberalism on the political and sociodemographic transition taking place in Mexico and the Socialist Republic of Vietnam, taking into account the similarities and differences between the two countries? The transformation of the State and society in Mexico and Vietnam took off when the countries began to implement neoliberal policies in the 1980s, the modus operandi being a reduced role for the State and a weaker relationship with the economy in both countries. Neoliberalism has also had an impact on the social sphere, evident in an analysis of the demographic transition, creating sociodemographic gaps between population groups, as well as in the realm of education and labor, and even more so for the indigenous population.
\end{abstract}

Key Words: Neoliberal policies, State crisis, sociodemographic transition, education, indigenous population. 


\section{LES PROBLÈMES DE LA TRANSITION ÉCONOMIQUE ET SOCIO-DÉMOGRA- PHIQUE DU MEXIQUE ET VIETNAM \\ Résumé}

Quels sont les impacts du néolibéralisme sur la transition politique et socio-démographiques du Mexique et la République socialiste du Vietnam, en tenant compte des similitudes et des différences entre les deux pays? La dynamique de la transformation de l'Etat et de la société mexicaine et vietnamienne ont accéléré le processus de la mise en œuvre des politiques néolibérales au cours des années quatre-vingt; le modus operandi de l'affaiblissement du rôle de l'Etat et sa relation avec l'économie des deux pays. L'analyse de la transition démographique montre que le Néolibéralisme a également eu un impact sur le domaine social, en particulier la création d'écarts démographiques entre les groupes de population, et aussi dans le domaine de l'éducation et du travail, spécialement dans la population indigène.

Mots clés: les politiques néolibérales, la crise de l'Etat, la transition démographiques, l'éducation, la population indigène.

\section{PROBLEMAS NA TRANSIÇÁO ECONÓMICA E SÓCIO-DEMOGRÁFICA NO MÉXICO E VIETNÁ \\ Resumo}

Quais são os impactos do neoliberalismo na transição política e sócio-demográfica do México e da República Socialista do Vietnã, tendo em conta as semelhanças e diferenças entre os dois países? A dinâmica da transformação do Estado e da sociedade no México e no Vietnã acelerou o processo de implementação das políticas neoliberais a partir da década de oitenta, modus operandi da diminuição do papel do Estado e sua relaçáo com a economia de ambos países. $\mathrm{O}$ neoliberalismo também teve um impacto sobre a esfera social, ao analisar a transição demográfica, criando vazios demográficos entre grupos populacionais, também na esfera educacional e de trabalho, especialmente na população indígena.

Palavras-chave: políticas neoliberais, crise de Estado, transição sócio-demográfica, educação, população indígena.

墨西哥和越南经济及社会人口结构转型中的问题

卢卡斯・萨尔内基

摘要

本文综合考虑墨西哥和越南两国的相似性及差异, 探讨新自由主义在政治 和社会人口转型中的影响。随着上世纪 80 年代新自由主义政策的实施, 墨 西哥和越南两国的国家及社会转型快速发展。新自由主义政策减少了政府 在经济中的作用。新自由主义在社会领域也有影响, 本文在分析人口转型 时发现新自由主义导致了不同社会群体之间的社会及人口差距。此外, 新 自由主义在教育和劳动领域, 甚至在土著居民人口方面也有影响。 关键词：新自由主义政策，国家危机，社会人口转型，教育，土著居民 
El pueblo de Vietnam ha ganado ya un lugar prominente en la historia contemporánea y sus luchas continuan sin tregua. A ese pueblo heroico, valiente $y$ revolucionario, que construye una sociedad socialista en difíciles condiciones, pero con el entusiasmo de quienes tienen una fe inquebrantable en el futuro, dedico estas modestas páginas, fruto de un viaje inolvidable y fructifero.

Ángel Bassols Batalla (1981: 8)

\section{INTRODUCCIÓN}

A partir de finales de los años ochenta, la dinámica de la transformación del Estado y la sociedad en México y Vietnam se aceleró con el proceso de implementación de políticas neoliberales. En México, se introdujo el ajuste estructural y las reformas del mercado durante la administración del presidente Miguel de la Madrid (1982-1988). Fue en la misma década, cuando en Vietnam se implementaron reformas orientadas hacia la apertura del mercado, denominadas como la política Đổi Mới, término que significa la "renovación", relacionadas con la crítica interna del VI Congreso Nacional del Partido Comunista celebrado en diciembre de 1986.

El objetivo del artículo es analizar, por un lado, el impacto del neoliberalismo en la transformación política, es decir, la posición del Estado en relación con las fuerzas mercantiles; y, por otro lado, el impacto del neoliberalismo en la sociedad, analizando las transiciones sociodemográficas, así como la educación y las relaciones laborales en ambos países.

El común denominador para ambas naciones son los procesos negativos que acompañan las transformaciones sociodemográficas durante las décadas del neoliberalismo, como son: la exclusión social, las desigualdades, la precariedad laboral, profundizando las brechas dentro de los grupos poblacionales y aún más entre la población indígena. Por si fuera poco, el impacto del neoliberalismo se observa en la esfera social: en la transición sociodemográfica, en la educación y en las relaciones laborales. El Estado no interviene en el apoyo de los grupos excluidos, no actúa para disminuir las desigualdades educativas, manteniendo las condiciones precarias de una vida laboral de miseria para muchos.

El presente trabajo se dividió en cinco partes; además de la introducción y las reflexiones finales, se analizó la transformación política, después se abordó el impacto del neoliberalismo en la transición sociodemográfica, para finalmente, presentar el apartado sobre el impacto del neoliberalismo en la población indígena. 


\section{EL ESTADO Y EL PROCESO DE LA TRANSFORMACIÓN NEOLIBERAL}

En México y Vietnam dio inicio el periodo de transformación neoliberal desde los años ochenta. Este cambio tuvo impacto para la concepción del Estado que se vio reflejada en las reformas constitucionales de ambos países. En lo que se refiere a el caso mexicano, después de tres siglos de Virreinato y relaciones de dependencia con la Corona española, desde el siglo xix comenzó, esencialmente asimétrica, la inevitable asociación (inevitable partnership) con Estados Unidos (Smith, 2000). El impacto de ambas influencias, virreinal y norteamericana, se encuentra reflejada en la Constitución política de 1917. Además de las influencias en la construcción del Poder Ejecutivo, Legislativo y Judicial, la Constitución estableció el régimen de la protección de los derechos sociales. Se trata, pues, de los derechos a la educación laica (artículo $3^{\circ}$ ), derecho a la salud (artículo $5^{\circ}$ ), derecho a la tierra (artículo 27) y derecho al trabajo (artículo 123), principalmente.

Se establecieron así, los marcos visibles de la fuerte presencia del Estado en el seno de la sociedad y la economía. El espíritu de la Constitución marca la posición central del Estado. De acuerdo con el artículo 25: "El Estado Federal dará lugar al desarrollo nacional que será integral y sostenible [...]. Teniendo en cuenta la equidad social y la productividad como las directrices del sector público deberá ayudar y promover las empresas, tanto en el sector privado y el sector social de la economía".

En lo que se refiere al artículo $3^{\circ}$, éste fue el más discutido durante el Constituyente de 1916-1917. Cabe destacar que el entonces presidente Venustiano Carranza estuvo solo una vez presente durante el debate, justo durante la sesión del 13 de diciembre de 1916 cuando se discutió el artículo 3º, en el que se estableció el principio de la educación libre y laica como conductas del Estado. Respecto al artículo 27, el asunto de la tierra fue uno de los más importantes durante la Revolución y después durante el Congreso Constituyente. Para Marván Laborde existen cinco puntos fundamentales dentro de este artículo:

primero, la definición de propiedad de tierras y aguas corresponde a la Nación; segundo, entre los bienes de la Nación se encuentran los minerales del subsuelo lo que tiene antecedente en las Reales Ordenanzas de la Nueva Espańa de 1783; tercero, estos bienes de subsuelo no pertenecen a la propiedad particular y la explotación estará sujeta a concesión; cuarto, se establecieron restricciones y prohibiciones específicas para adquirir las tierras; quinto, se definieron las decisiones agrarias en cuanto al reparto a los pueblos (D.D., 2005: 1002-1005). 
Con base en el artículo 27 comenzó el reparto de las tierras. La fuerte presencia del Estado marcó los pasos hacia la repartición. Respecto al artículo 123, se estableció el derecho al trabajo, junto con el derecho de huelga para los obreros; se habló sobre el digno salario mínimo.

A lo largo del proceso de la transformación neoliberal, cambiaron los artículos respecto a los derechos sociales. Cabe destacar que la Constitución mexicana, con más de 600 enmiendas, es una de las más antiguas constituciones entre los países latinoamericanos y del Caribe.

La transformación de la centralidad del Estado hasta el proceso de su desaparición ocurrió a partir de los años ochenta y culminó en los noventa. La reforma al artículo 27 de la Constitución comenzó durante la administración del presidente Carlos Salinas de Gortari (1989-1994) en 1992. Con esta enmienda se terminó con el ejido y los propietarios colectivos del terreno podían vender las parcelas. La segunda fase se relaciona con cambios constitucionales durante la administración del actual mandatario Enrique Peña Nieto (20132018). Se introdujo el derecho de otorgar las concesiones para la explotación y extracción de hidrocarburos, "el uso o el aprovechamiento de los recursos de que se trata, por los particulares o por sociedades constituidas conforme a las leyes mexicanas, no podrá realizarse sino mediante concesiones" (artículo 27). Cuyo propósito es, se señala, obtener más ingresos para el Estado:

Con el propósito de obtener ingresos para el Estado que contribuyan al desarrollo de largo plazo de la Nación, ésta llevará a cabo las actividades de exploración y extracción del petróleo y demás hidrocarburos mediante asignaciones a empresas productivas del Estado o a través de contratos con éstas o con particulares, en los términos de la Ley Reglamentaria. Para cumplir con el objeto de dichas asignaciones o contratos las empresas productivas del Estado podrán contratar con particulares (artículo 27, párrafo adicionado 20 de diciembre de 2013).

El caso de la República Socialista de Vietnam, igual que el caso mexicano, muestra la transformación del orden jurídico a partir de los años ochenta. Históricamente, Vietnam se formó como Estado tomando en cuenta las relaciones con su gran vecino, China. Las relaciones vietnamitas con las dinastías feudales chinas se basaron en la investidura, que comenzó en el siglo x. De acuerdo con My Hanh: "para Vietnam, cuya frontera se encontraba junto a la Gran China feudal y que solía ser invadido por China desde hace miles de años, la investidura siempre se utilizó como una forma diplomática para mantener la amistad con este vecino fuerte" (My Hanh, 2016: 17). 
Después, en el siglo XIx comenzó la conquista francesa que se prolongó hasta la segunda mitad del siglo xx. Así, "la lucha de Vietnam fue ofensiva. Esa característica diferencía a los vietnamitas de aquellos que tenían que enfrentar a los conquistadores españoles en América Latina" (Czarnecki, 2011: 208). Bassols Batalla señalaba que "cuando se habla de Vietnam, debe por tanto considerárselo como un país socialista que tiene todas las posibilidades otorgadas por la creación de las estructuras socioeconómicas, cuanto como un país subdesarrollado, con todo lo que ello supone de dificultades, supervivencias y obstáculos" (1981: 58).

En 1946 se estableció la primera Constitución de la República Socialista de Vietnam, sin embargo, la guerra y la confrontación con la Francia imperialista y después con Estados Unidos duraron hasta los años setenta.

Además de la Constitución de 1946, en Vietnam se promulgaron las cuatro constituciones de 1959, 1980, 1992 y 2013. Como en el caso de México, los cambios constitucionales a partir de los años ochenta significaron la apertura al sistema del libre mercado (política de Đổi Mới, la "renovación").

En 1986, en el VI Congreso Nacional del Partido Comunista de Vietnam, se reconocieron "serios errores" en la política del Estado y el Partido se comprometió a implementar las reformas de la renovación, entre otras, en la esfera de la agroindustria: "el derecho al trabajo y el acceso al capital, así como el uso de las tierras a largo plazo (incluyendo bosques y las superficies de agua) por las comunidades rurales" (Xuan Nam, 2001: 103). Los campesinos ya no tuvieron la obligación de vender los productos al Estado a precios por él estipulados, sino que comenzaron a venderlos en el libre mercado.

En el preámbulo a la Constitución de 1992 se señaló que "desde 1986 se ha llevado a cabo un proceso de toda la ronda de la reforma y la renovación iniciada por el Sexto Congreso del Partido que ha logrado importantes logros iniciales. La Asamblea Nacional ha decidido revisar la Constitución de 1980 con el fin de satisfacer las exigencias de las nuevas circunstancias y tareas". Después, en 2001, se implementaron las enmiendas importantes que se refieran al libre mercado, como se señala en el artículo 15:

El Estado adopta políticas consistentes en el desarrollo de una economía de mercado socialista. La estructura multisectorial de la economía con tipos diversificados de producción y organización empresarial se basa en la propiedad de todo el pueblo junto a la propiedad colectiva y privada, de los cuales los dos primeros y el segundo son la piedra angular. 
La Constitución vietnamita a partir de 2013 muestra una especie de híbrido con respecto al papel del Estado en la economía. Estas interacciones entre el libre mercado y la economía del Estado socialista jugaron un papel crucial en los tiempos modernos. De acuerdo con el artículo 51 de su Carta Magna: "1. La economía vietnamita es economía de mercado socialista, con variadas formas de propiedad y los sectores económicos; la economía del Estado desempeña el papel dominante".

Por un lado, el Estado tiene la posición dominante, pero por el otro, de acuerdo con el artículo 51 punto 3: "El Estado promoverá y creará las condiciones para la gente de negocios, empresas u otros individuos, organizaciones para llevar a cabo la inversión, la producción o actividad; y desarrollar ramas económicas de manera sostenible con el fin de contribuir a la construcción nacional". En el artículo 52, la Constitución estipula: "El Estado debe desarrollar y mejorar las instituciones económicas, regular la economía sobre la base del respeto a las reglas del mercado".

En conclusión, en México y Vietnam se observan cambios en los ámbitos jurídicos y políticos desde los años ochenta. La transformación neoliberal tuvo impacto en la disminución del papel del Estado, dejando espacio para la liberalización, la privatización y la apertura al libre mercado, principalmente. El neoliberalismo debilitó al Estado en ambos países, entonces habría que preguntarse si ¡̨el neoliberalismo también debilitó a la sociedad?

\section{EL NEOLIBERALISMO Y LA TRANSFORMACIÓN SOCIODEMOGRÁFICA}

De acuerdo a las fuentes nacionales, el Instituto Nacional de Estadística y Geografía (INEGI), en México para el año 2013 había 118395054 habitantes (INEGI, 2016), y de acuerdo con la Oficina General de la Estadística de Vietnam, para el año 2013 había 89759500 personas (Gso, 2016). Cabe destacar que las fuentes nacionales e internacionales se diferencian significativamente. Según los datos de las Naciones Unidas (Profiles of Ageing, 2015), para el año 2013, a México corresponden 127017000 habitantes contra los 93448000 en Vietnam (véase gráfica 1). En el caso de México, la diferencia es significativa y resulta ser casi de 10 millones de habitantes, mientras que en Vietnam es de 4 millones. Estas son las diferencias tomando en cuenta las estadísticas nacionales e internacionales respecto a la población de ambos países. 
Gráfica 1. La población de México y Vietnam según datos de las Naciones Unidas

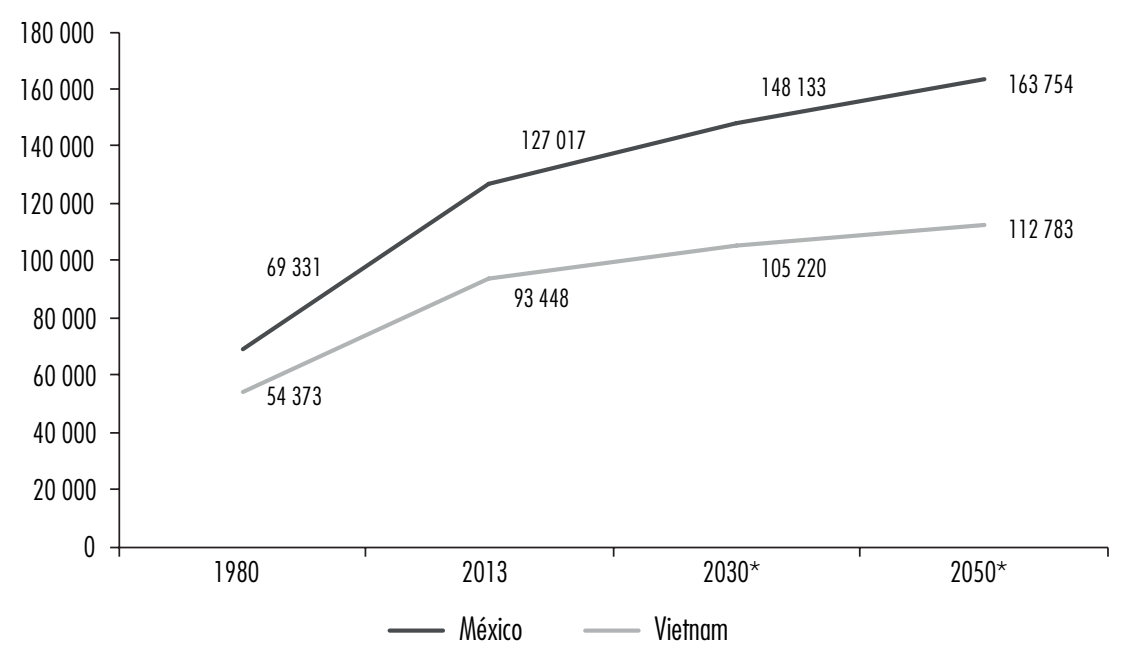

* Años 2030 y 2050 son una proyección.

Fuente: elaboración propia con base en Profiles of Ageing 2015, Naciones Unidas.

Respecto a la población de 0-14 años y a la población de 60 años y más, se observan los cambios en la disminución de la población joven y el crecimiento de la población de 60 años y más, como muestran los cuadros 1 y 2, según fuentes nacionales. Para el año 2009, el porcentaje de la población de 0-14 años había disminuido durante las últimas décadas, de 36.3, en 1989, a 29.8, en 2010, en el caso de México, y de 35, en 1989, a 23.2, en 2009, en el caso de Vietnam (véase cuadro 1).

Al mismo tiempo, crece la población adulta mayor de 60 y más en ambos países. Para el 2010, el porcentaje de la población de 60 años y más es mayor en México (9.2\%) que en Vietnam (8.6\%), como muestra el cuadro 2.

El cuadro 3 muestra la transformación de la población de 0-14 años y la población de 65 y más años con base en las cifras de las Naciones Unidas. Mientras en 1980, en el caso de México, la población de 0-14 representaba el 45.3\%, para el 2013 era de $27.6 \%$.

En el caso de Vietnam, de manera parecida, en 1980, la población de 0-14 representaba el $40.9 \%$ y para 2013 era de $23.1 \%$.

En cuanto a la población de las personas adultas mayores de 65 y más, Vietnam tiene una población adulta mayor superior que México: 3.2 y 4.6\%, en 1980, para México y Vietnam, respectivamente; y 6.5 y $6.7 \%$, en 2013, respectivamente. 
Cuadro 1. Porcentaje de la población 0-14 en México y Vietnam

\begin{tabular}{lccc}
\hline México & 1990 & 2000 & 2010 \\
\hline $0-14$ & 36.3 & 33.2 & 29.8 \\
\hline Vietnam & 1989 & 1999 & 2009 \\
\hline $0-14$ & 35 & 29.6 & 23.2 \\
\hline
\end{tabular}

Fuente: elaboración propia con datos de IPUMS. Minnesota Population Center. Minneapolis: Universidad de Minnesota, 2015.

Cuadro 2. Porcentaje de la población 60 y más en México y Vietnam

\begin{tabular}{lccc}
\hline México & 1990 & 2000 & 2010 \\
\hline 60 y más & 6.1 & 7.1 & 9.2 \\
\hline Vietnam & 1989 & 1999 & 2009 \\
\hline 60 y más & 6.2 & 7.4 & 8.6 \\
\hline
\end{tabular}

Fuente: elaboración propia con datos de IPUMS. Minnesota Population Center. Minneapolis: Universidad de Minnesota, 2015.

Cuadro 3. Porcentaje de la población 0-14 y 65 y más para México y Vietnam según los datos de las Naciones Unidas

\begin{tabular}{lrrrc}
\hline Edad/Año & 1980 & 2013 & $2030^{*}$ & $2050^{*}$ \\
\hline 0-14 México & 45.3 & 27.6 & 22.1 & 16.9 \\
$65+$ México & 3.2 & 6.5 & 10.4 & 18.9 \\
0-14 Vietnam & 40.9 & 23.1 & 20.2 & 17.2 \\
65+ Vietnam & 4.6 & 6.7 & 12.4 & 21 \\
\hline
\end{tabular}

* Años 2030 y 2050 son una proyección.

Fuente: elaboración propia con base de Profiles of Ageing 2015, Naciones Unidas. 


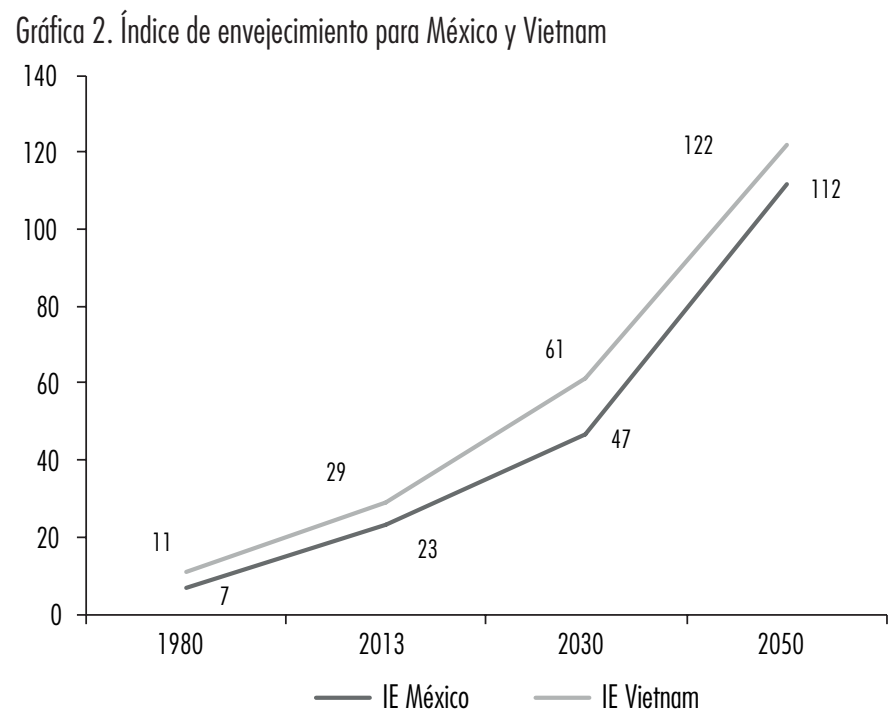

Fuente: elaboración propia con base en Profiles of Ageing 2015, Naciones Unidas.

La gráfica 2 muestra el índice de envejecimiento, que representa la cantidad de personas adultas mayores, de 65 y más ańos por cada 100 niños y jóvenes; este índice se calcula con la división del número de personas de 65 años y más entre el número de personas de 0-14 años.

Ambos países muestran un índice de envejecimiento parecido, sin embargo, para el 2013, Vietnam presentó un índice de envejecimiento mayor. Hay 29 personas adultas mayores de 65 y más por cada 100 nińos y jóvenes de 0-14 años. En el caso de México, la cifra fue de 23. Esto porque, como muestra el cuadro 1, la dinámica del cambio de población de 0-14 años es mucho mayor en el caso de Vietnam que en México. En Vietnam, la población de 0-14 años disminuye de $35 \%$, en 1989 , a $29.6 \%$, en 1999 , y a $23.2 \%$ para 2009 . En el caso de México, la población de 0-14 años disminuye de 36.3\%, en 1990, a $33.2 \%$, para 2000 , y $29.8 \%$ en 2010 .

Basándose en los censos nacionales de Vietnam (1989, 1999 y 2009) y de México (1990, 2000 y 2010), se aprecia la transformación en la dinámica de la población en ambos países (véase gráfica 3).

En el caso de Vietnam, en 1989, el número de personas de 0-14 años fue mayor que en los otros grupos de edad, pero en 1999 hubo una gran reducción de personas de 0-4 años. En la pirámide de edades a partir de 2009, hay un número significativo de personas de 15-29 años. En el caso de México, se 
observa la disminución de la población joven y crecimiento de la población adulta mayor. Cabe destacar que, en ambos países, la población de 60 años y más son en su mayoría mujeres.

Basándose en los datos de las Naciones Unidas, México y Vietnam tienen una posición similar con respecto al porcentaje de la población adulta mayor de 60 años o más, como se muestra en el cuadro 4.

Cuadro 4. La clasificación en función del porcentaje de población mayor de 60 años 0 más en 2013

\begin{tabular}{lcc}
\hline País & Población de 60 y más & Lugar \\
\hline Japón & 32.0 & 1 \\
Italia & 26.9 & 2 \\
Alemania & 26.8 & 3 \\
Vietnam & 9.5 & 92 \\
México & 9.3 & 94 \\
\hline Fuente: elaboración propia con base en los datos de Naciones Unidas (2013: 94).
\end{tabular}

Para concluir este apartado, se demuestra que el proceso del cambio estructural en los hogares vietnamitas y mexicanos es resultado de las múltiples transformaciones sociales, culturales y económicas. Para ambos países, la tasa de nacimiento tiende a bajar y viene acompañada con la reducción del número de los miembros de la familia (Long, 2013: 33-34). Se observa el envejecimiento de la población. Según el estudio de Second National Survey Assessment of Vietnamese Youth (SAVY2) en 2009, los jóvenes vietnamitas tienden a una "elección de estar solo" y una actitud positiva de la "mujer siendo no esposa, pero teniendo hijos" (Huu Minh y Thi Hong, 2015: 27). Sin embargo, estos cambios vienen acompañados de la polarización y las desigualdades dentro de los grupos poblacionales, la precariedad laboral de los grupos vulnerables como jóvenes, mujeres y población indígena, principalmente (Navarrete, 2012). 


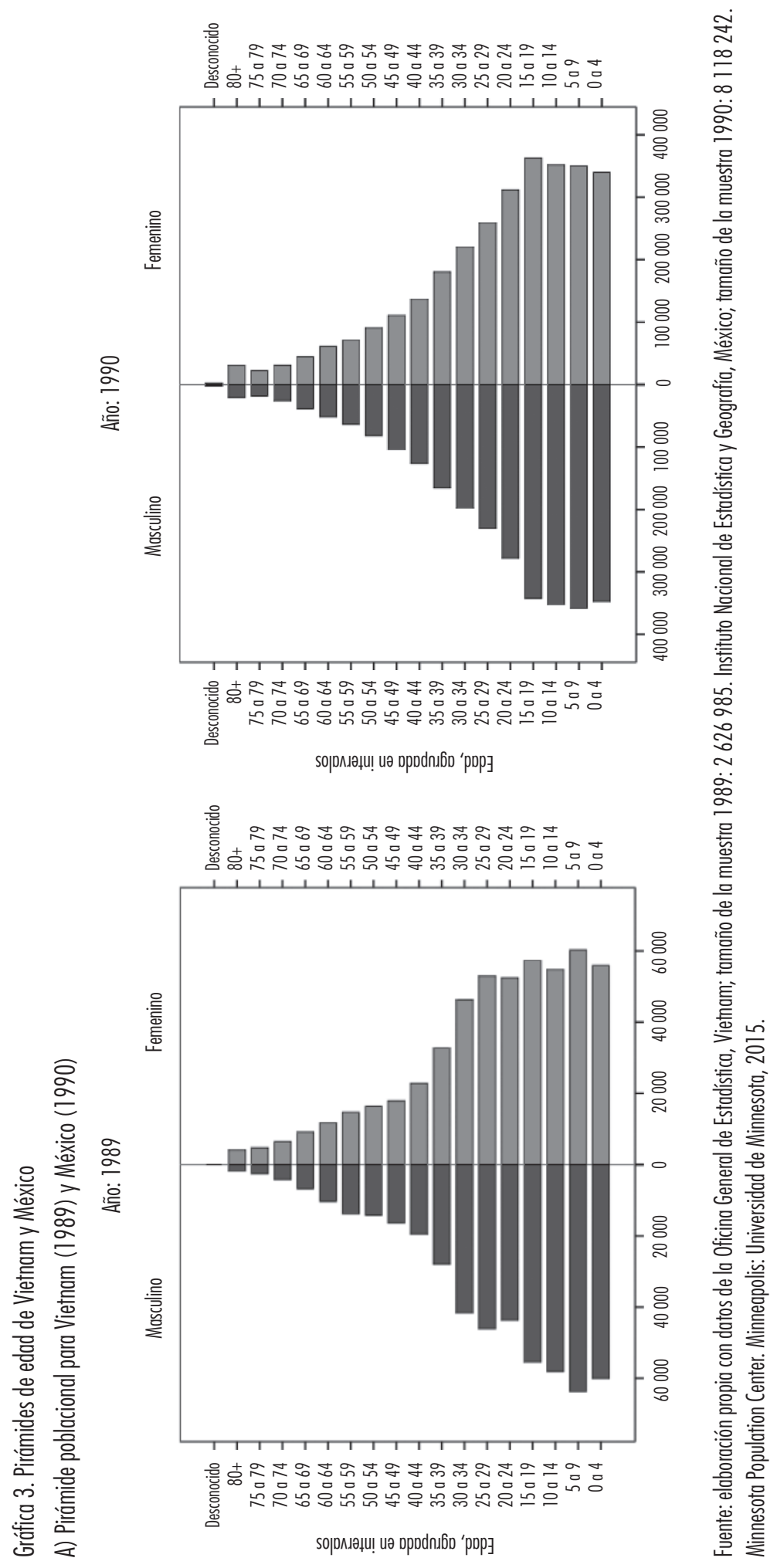




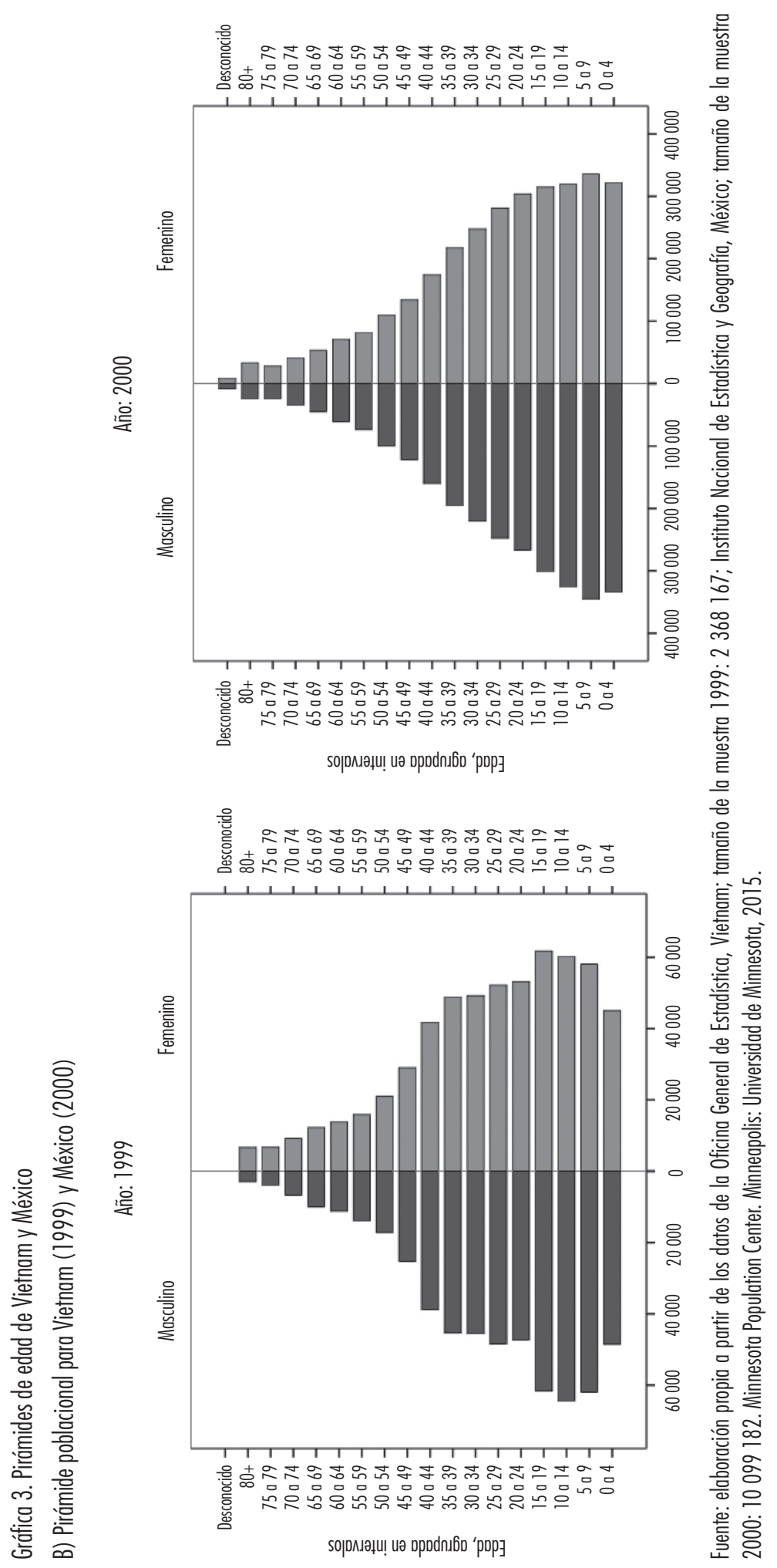




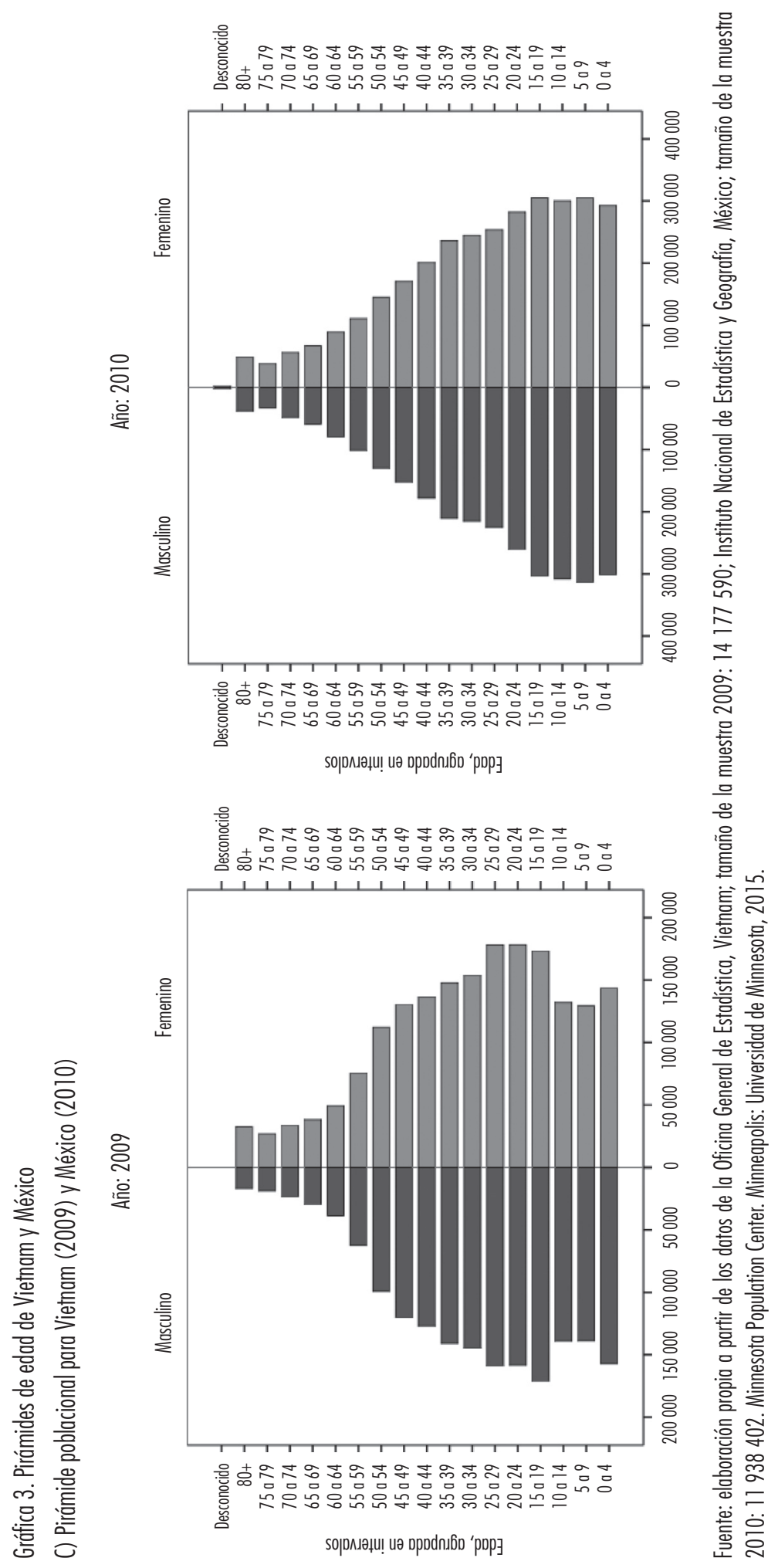




\section{¿Cuál es el impacto del neoliberalismo en la esfera de la educación?}

El analfabetismo, a pesar de las transformaciones, todavía sigue presente, prácticamente sin cambio alguno en el caso México, como muestra el cuadro 5. El porcentaje de las personas que lee y escribe en Vietnam creció desde el 81\% (1989) hasta el $85.1 \%$ (1999) y el 86.9\% (2009); mientras que en el caso de México, el porcentaje de la gente que sabe leer y escribir se incrementó desde 78.7\% (1990) hasta el 79.7\% (2000) y el 80.5\% (2010).

La dinámica del cambio hacia la alfabetización es mayor en Vietnam que en México; en el caso de Vietnam, subió sobre el 5.9\% entre 1989 y 2009; en el caso de México, sobre el 1.8\% entre 1990 y 2010. Para el año 2010, el 8.8\% eran analfabetas en México, mientras que el 4.7\% en Vietnam en 2009 (véase cuadro 5). El neoliberalismo acentuó el rezago educativo. Las brechas inter e intrageneracionales siguen creciendo donde, a pesar de la ampliación de la cobertura educativa, "México es aún una sociedad con amplias desigualdades educativas” (Solís, 2015: 90).

Cuadro 5. El porcentaje de analfabetismo en México y Vietnam

\begin{tabular}{lrrrrrr}
\cline { 2 - 6 } & Vietnam & México & Vietnam & México & Vietnam & México \\
\cline { 2 - 6 } & 1989 & 1990 & 1999 & 2000 & 2009 & 2010 \\
\hline No se encuentra en el universo & 11.5 & 11.9 & 8.2 & 11 & 8.3 & 9.7 \\
No, analfabeta & 7.4 & 9 & 6.6 & 9.1 & 4.7 & 8.8 \\
Sí, lee y escribe & 81 & 78.7 & 85.1 & 79.7 & 86.9 & 80.5 \\
Desconocido & 0.1 & 0.4 & 0.1 & 0.2 & 0.1 & 0.9 \\
\hline
\end{tabular}

Fuente: elaboración propia con datos de IPUMS. Minnesota Population Center. Minneapolis: Universidad de Minnesota, 2015.

El cuadro 6 muestra la comparación entre los años de escolarización en ambos países. Se observan los cambios en la duración de los años de educación, desde 1989/1990 hasta 2009/2010.

Cabe destacar que para ambos países aumentó el número de personas que dedican más años a la escuela. Sin embargo, se observa también el aumento de las personas que no tienen ningún tipo de educación escolarizada o que tienen solamente educación preescolar; este dato es más grande para México que para 
Vietnam; en el caso de Vietnam, el porcentaje de estas personas sube de 9.6\% en 1989, a $11.5 \%$, en 2009, y en el caso de México, crece de $13.1 \%$ en 1990 a $14.1 \%$ en 2010 .

El porcentaje de personas que cursan de 1 hasta 4 ańos en la escuela crece con el transcurso de tiempo. Entre 5 y 8 años baja, en el caso de México, y en el caso de Vietnam, sube el porcentaje de las personas de 5-7 años. Los años de escolarización suben, en el caso de México, entre 9 y 18 años. También en el caso de Vietnam, con un crecimiento de más del 100 por ciento para los años de escolarización de 9 años, de 5.3\%, en 1989, a 12.8\%, en 2009. En el caso de México, la dinámica del crecimiento más significativa es de 12 años, cuando subió el porcentaje de 3.2\%, en 1990, a 8.5\%, en 2010 .

$\mathrm{Al}$ analizar el cuadro 6, se aprecia el crecimiento de las brechas entre las personas que dedican tiempo a los años de educación. Por un lado, crece el número de las personas sin educación o solo con la educación preescolar en ambos países. Sin embargo, en el caso de México este porcentaje es mayor que en el caso de Vietnam. Por el otro, crece el porcentaje de las personas con una escolarización mayor a una década. Las brechas siguen aumentando en tiempos de las políticas neoliberales.

Finalmente, después del análisis del impacto del neoliberalismo a la transición demográfica y a la educación, cabe preguntarse sobre el impacto de éste en la esfera laboral.

Con base en los datos, accesibles sólo para 2009 y 2010, en ambos países, la cuarta parte de la población son trabajadores asalariados, es decir, 25\%, como muestra el cuadro 7. Se aprecia que los trabajadores por cuenta propia en México ocupan el $10.8 \%$ de las personas, mientras en Vietnam la cifra es el doble. También, se aprecia el alto número de personas que no están en el universo, el número es más grande para México (62.1\%) que para Vietnam $(49.7 \%)$, seguramente un resultado de la alta informalidad en ambos países (Hanson, 2010; Lincoln, 2008). Además, se subraya que existen las condiciones laborales muy pobres y desventajosas; en el caso de Vietnam, "la luz inadecuada, ruido, superpoblación, calor y otras deficiencias son frecuentes. Los trabajadores trabajan normalmente muchas horas sin descanso, tampoco sin estándares de seguridad para protegerlos" (Hong Xoan, 2015: 35). El gobierno comunista, para atraer la inversión extranjera, "ha intentado competir con otras naciones en la región en términos de ofrecer mano de obra barata" (Hong Xoan, 2015: 35); en el caso de México, la situación resulta pareci$\mathrm{da}$ con las condiciones laborales denigrantes, especialmente en maquiladoras donde los trabajadores tienen bajos salarios y laboran muchas horas (Navarrete y Aragón-Durand, 2011). 
Cuadro 6. Los años de educación en Vietnam y México

\begin{tabular}{lrrrrrrr} 
& \multicolumn{3}{c}{ Vietnam } & & & México \\
\cline { 2 - 4 } & 1989 & 1999 & 2009 & & 1990 & 2000 & 2010 \\
\hline Ninguno o preescolar & 9.6 & 8.3 & 11.5 & & 13.1 & 9.9 & 14.1 \\
1 año & 3.8 & 2.9 & 2 & & 3.9 & 4 & 3.5 \\
2 años & 5 & 4.1 & 3.2 & & 5.2 & 4.7 & 4.4 \\
3 años & 6 & 5.2 & 3.8 & & 6.4 & 5.7 & 5.3 \\
4 años & 5.3 & 5.3 & 4 & & 4.8 & 4.1 & 3.6 \\
5 años & 8.5 & 8.1 & 9 & & 4.1 & 3.6 & 3.3 \\
6 años & 5 & 5.4 & 5.1 & & 16.4 & 14.2 & 13.1 \\
7 años & 5.2 & 6.5 & 5.5 & & 3 & 2.9 & 2.7 \\
8 años & 11.9 & 11.8 & 4.4 & & 3.6 & 3.5 & 3.4 \\
9 años & 5.3 & 6.8 & 12.8 & & 13.7 & 13.2 & 15.5 \\
10 años & 3.6 & 3.6 & 4.2 & & 1.8 & 2.2 & 2.2 \\
11 años & 3.4 & 3.6 & 8 & & 2.2 & 2.5 & 2.7 \\
12 años & 12.4 & 9.1 & 11.6 & & 3.2 & 7.2 & 8.5 \\
13 años & 3 & & 1.1 & & 0.7 & 1.3 & 1.1 \\
14 años & & 1.1 & 0.9 & 0.7 & 0.8 & 1 \\
15 años & & & 2.4 & 0.8 & 1.1 & 1.8 \\
16 años & & 3.3 & 4.8 & 1.4 & 2.8 & 3.5 \\
17 años & & & 1.8 & 1.4 & 2.3 & 2.3 \\
18 años y más & 0 & 0.1 & 0.6 & & 0.9 & 0.5 & 0.9 \\
\hline
\end{tabular}

Fuente: elaboración propia con datos de IPUMS. Minnesota Population Center. Minneapolis: Universidad de Minnesota, 2015. 
Cuadro 7. El porcentaje de la población según su situación en el empleo

\begin{tabular}{lcc}
\cline { 2 - 3 } & México & Vietnam \\
\cline { 2 - 3 } & 2010 & 2009 \\
\hline No está en el universo & 62.1 & 49.7 \\
Trabaiadores por cuenta propia & 10.8 & 19.9 \\
Trabajadores asalariados & 25.2 & 25.1 \\
Trabajador sin pago & 1.1 & 5.1 \\
Desconocido & 0.8 & 0.1 \\
\hline
\end{tabular}

Fuente: elaboración propia con datos de IPUMS. Minnesota Population Center. Minneapolis: Universidad de Minnesota, 2015.

Para concluir, la disminución de la población de 0-14 años y el crecimiento de la población de 60 años y más muestra el proceso acelerado de envejecimiento que, a su vez, se relaciona con la insuficiencia del sistema de protección social en México y Vietnam. Según Cristina Gomes: "Entre 1990 y 2000, el número de personas mayores de 60 ańos de edad aumentó de cerca de 5 millones a cerca de 7 millones, o del 6 al 7\% de la población total de México" (Gomes, 2007: 546). En México, el apoyo institucional, como las pensiones es escasa. Ese es el contexto de la importancia de las redes familiares y de solidaridad para apoyar a las personas mayores, es decir, el apoyo intergeneracional:

Las diferentes generaciones intercambian el dinero, servicios, regalos, cuidado y otros tipos de apoyo, de acuerdo con los roles de género. Los hombres reproducen su papel de proveedor económico y dan recursos monetarios a los familiares. Tener dinero depende de tener un sueldo y esto es dos veces más frecuente entre los hombres de edad avanzada que entre las mujeres de edad avanzada, y el doble de hombres reciben una pensión que las mujeres. Los que están en una situación económica en desventajas, por consiguiente, serían "dependientes" de sus familiares (Gomes, 2007: 552).

A la misma conclusión llegaron los autores del estudio de una muestra nacionalmente representativa de 2.376 hombres de 60 y más ańos de edad en 1994: "las tres cuartas partes de los hombres encuestados recibieron apoyos 
en especie, doméstico, financiera o asistencia física en el mes anterior a la de conclusión 1994 encuesta" (De Vos et al., 2004: 23). La ayuda informal se extiende en las familias que "han desarrollado complejas redes de apoyo informal, que, además de la transferencia económica o las remesas también incluyen donaciones, regalos y servicios intercambiados entre familiares y amigos" (Gomes, 2007: 558).

En el caso de Vietnam, como en el caso mexicano, el sistema de protección es muy limitado y se caracteriza por: "1) la administración fragmentada con varios ministerios, departamentos y organizaciones que proporcionan la protección; 2) una cobertura limitada a una pequeña proporción del sector público, y 3) se centró principalmente en las prioridades de los empleados estatales y los funcionarios públicos" (Nguyen Anh, 2014: 10). De ahí, la ayuda informal tiene la mayor importancia, donde se desarrollan las redes de apoyo por parte de la familia. Se subraya que, "la cobertura de la seguridad social y el sistema de pensiones se mantiene en su mayoría restringidas a una pequeña proporción de los empleados en el sector público o formal" (Nguyen Anh, 2014: 11).

$\mathrm{Al}$ analizar el impacto del neoliberalismo en la esfera sociodemográfica, educativa y laboral, cabe subrayar que existen los grupos vulnerables que sufren aún más condiciones de exclusión y precariedad. El tema se abordará a continuación.

\section{EL NEOLIBERALISMO Y LA POBLACIÓN INDÍGENA}

En el neoliberalismo, las competitivas fuerzas del mercado excluyen los grupos vulnerables, ya que el Estado no funge como fuerza reguladora. ¿Acaso esto ocurre con la población indígena de ambos países?

México, al igual que la República Socialista de Vietnam, tiene el mismo número considerable de grupos de la población indígena, entre 50 y 60 (véase cuadro 9). Los cambios sociodemográficos de los hogares en México y Vietnam resultan tener diferentes características dentro de los grupos poblacionales. La población indígena en ambos países tiene mayores desventajas y sufren mayores precariedades estructurales. Cabe destacar que en México se distingue a la población indígena por la lengua, mientras que, en Vietnam, se distingue por las minorías étnicas.

Los Kinh (Viet) ocupan entre 80 y $90 \%$ de la población total y los 10 grupos indígenas más grandes son, en el caso de Vietnam: Hoa $(0.81 \%)$, Tay $(0.79 \%)$, Kho Me $(0.35 \%)$, Nung $(0.34 \%)$, Thai $(0.30 \%)$, Muong $(0.14 \%)$, 
Dao (0.07\%), Gia Rai (0.07\%), Ra Na (0.07\%), Hmong (0.06\%). En el caso de México: náhuatl (3.7\%), mixteco (2.2\%), tzotzil (2.0\%), zapoteco $(1.9 \%)$, maya $(1.7 \%)$, tzeltal $(1.6 \%)$, mazateco $(0.9 \%)$, totonaca $(0.9 \%)$, tlapaneco $(0.8 \%)$, mixe $(0.5 \%)$ (como se muestra en el cuadro 9$)$.

En el caso de Vietnam, el resultado del censo de la población en 2009 mostró que entre los seis grupos étnicos más grandes hay diferencias de tamaño en los hogares; el grupo Kinh tiene el número más pequeño de miembros por hogar, promedio 4.3 personas, mientras que el grupo Hmong tiene el número más grande, 5.3 personas por hogar (Long, 2013: 25).

Ambos países presentan similitudes respecto al tamaño de la población indígena. El número de la población de etnias es difícil de considerar: en caso de Vietnam, según las fuentes no oficiales, hay entre y 10 y 15\% (Czarnecki, 2011: 200). Para el año 2009, los datos oficiales muestran que la población Kinh ocupa $91.3 \%$ y el resto, alrededor de $9 \%$ lo ocupaban diferentes minorías étnicas (véase cuadro 9).

En el caso de México, el porcentaje de la población que solo habla español y no habla ninguna lengua indígena disminuye de $80.9 \%$ en 1990 a $73.7 \%$ en 2010, como muestra el cuadro 8 . Sube casi el triple el porcentaje de personas que habla alguna lengua indígena y español, de 5.2\% en 1990 a $14.4 \%$ en 2010; también crece el porcentaje de la población que habla solamente la lengua indígena, de $1 \%$ en 1990 a $5.2 \%$ en 2010 .

Cuadro 8. El porcentaje de la población hablante lengua indígena, México 2010

\begin{tabular}{lrcc}
\cline { 2 - 4 } & 1990 & 2000 & 2010 \\
\hline No está en el universo & 12.6 & 11.6 & 6.1 \\
Si, habla lengua indigena y español & 5.2 & 8.2 & 14.4 \\
Si, solo habla lengua indígena & 1.0 & 2.1 & 5.2 \\
No, no habla lengua indígena & 80.9 & 77.8 & 73.7 \\
Desconocido & 0.3 & 0.3 & 0.3 \\
Total & 100 & 100 & 100 \\
\hline
\end{tabular}

Fuente: elaboración propia con datos de IPUMS. Minnesota Population Center. Minneapolis: Universidad de Minnesota, 2015. 
Según datos del Consejo Nacional de Evaluación de la Política de Desarrollo Social (CONEVAL), el 10\% de la población censada de origen indígena (10.8 millones) en 2000 subió al 16\% (18.1 millones) en 2010 (CONEVAL, 2014: 14). Se señala que "en México, la condición de pertenencia a un pueblo étnico originario constituye un factor no sólo de diferenciación, sino también de exclusión" (coneval, 2014: 120); donde, "prácticamente desde cualquier abordaje metodológico, la población con algún rasgo de pertenencia étnica se encuentra en mayores condiciones de precariedad que las personas que no los presentan. Son pobres entre los pobres" (CONEVAL, 2014: 144). El Estado no creó las políticas públicas igualitarias.

En el caso de Vietnam, parecido al caso de México, los grupos indígenas se tratan como "atrasados", como es el caso de los Hmong (Turner y Michaud, 2008: 160). Para los expertos del Banco Mundial, las fuentes de desigualdades étnicas en Vietnam se originan en "poca accesibilidad a la economía del mercado", entre otros factores (Van de Walle y Gunewardena, 2011: 203). Sin embargo, son las estructuras del capital transnacional y los grupos financieros internacionales, modus operandi del Estado nacional que excluyen los grupos vulnerables. Frente a los procesos de la exclusión, los indígenas se adaptan a través de las estrategias de sobrevivencia y se reinventan a sí mismos, tomando las oportunidades que surgen en cualquier momento y no en la elección racional o beneficio económico (Turner y Michaud, 2008). Tal es el caso de Chuy, mujer Hmong de 37 años, de Hau Thao, la localidad a 15 km de Sa Pa, la capital de la provincia Lao Cai: "Ni el gobierno, tampoco empresas privadas contribuyen en algo para las comunidades étnicas". ${ }^{1}$ La señora Chuy comenzó trabajar como una guía turística por cuenta propia para responder a las necesidades principales de su familia.

En conclusión, la situación de la población indígena presenta desventajas y precariedades estructurales en ambos países, pero a pesar de ello es la población que crece y transmite la enseñanza de la lengua indígena. Además, la población indígena emprende estrategias de adaptación en escenarios de exclusión.

\footnotetext{
Entrevista con Chuy, mujer Hmong, Sa Pa, provincia Lao Cai, Vietnam, enero de 2016.
} 
Lukasz Czarnecki

Cuadro 9. El porcentaje de la población indígena en Vietnam (2009) y México (2010)

\begin{tabular}{|c|c|c|c|c|c|}
\hline \multicolumn{3}{|c|}{ Vietnam } & \multicolumn{3}{|c|}{ México } \\
\hline & Frecuencia & Porcentaje & & Frecuencia & Porcentaje \\
\hline Kinh & 5266124 & 96.72 & Español solo & 8794833 & 73.7 \\
\hline Hoa & 44182 & 0.81 & Náhuatl & 441765 & 3.7 \\
\hline Tay & 43251 & 0.79 & Mixteco & 259305 & 2.2 \\
\hline Kho Me & 19127 & 0.35 & Tzotzil & 240013 & 2.0 \\
\hline Nung & 18548 & 0.34 & Zapoteco & 232676 & 1.9 \\
\hline Thai & 16433 & 0.30 & Maya & 198414 & 1.7 \\
\hline Muong & 7665 & 0.14 & Tzeltal & 194316 & 1.6 \\
\hline Dao & 4073 & 0.07 & Mazateco & 108170 & 0.9 \\
\hline Gia Rai & 3660 & 0.07 & Totonaca & 102495 & 0.9 \\
\hline $\mathrm{Ra} \mathrm{Na}$ & 3568 & 0.07 & Tlapaneco & 96130 & 0.8 \\
\hline Hmong & 3016 & 0.06 & Mixe & 59755 & 0.5 \\
\hline E De & 2140 & 0.04 & Chol & 47156 & 0.4 \\
\hline Xu Dang & 1934 & 0.04 & Chinanteco & 40405 & 0.3 \\
\hline Hre & 1566 & 0.03 & Otomí & 37721 & 0.3 \\
\hline Cham & 1360 & 0.02 & Zoque & 35009 & 0.3 \\
\hline San Div & 1192 & 0.02 & Chatino & 34677 & 0.3 \\
\hline Unknown & 945 & 0.02 & Amuzgo & 32007 & 0.3 \\
\hline Co Tu & 834 & 0.02 & Popoluca & 25854 & 0.2 \\
\hline San Chay & 701 & 0.01 & Huasteco & 24475 & 0.2 \\
\hline Giay & 693 & 0.01 & Tepehuano & 22788 & 0.2 \\
\hline $\mathrm{COH}_{\mathrm{O}}$ & 541 & 0.01 & Huichol & 22550 & 0.2 \\
\hline Gie Trieng & 474 & 0.01 & Purépecha & 17765 & 0.1 \\
\hline Ha Nhi & 392 & 0.01 & Cora & 17223 & 0.1 \\
\hline Bru-Van Kieu & 379 & 0.01 & Tarahumara & 13140 & 0.1 \\
\hline Tho & 280 & 0.01 & Cuicateco & 10415 & 0.1 \\
\hline
\end{tabular}


Problemáticas en la transición económica y sociodemográfica de México y Vietnam

\begin{tabular}{|c|c|c|c|c|c|}
\hline \multicolumn{3}{|c|}{ Vietnam } & \multicolumn{3}{|c|}{ México } \\
\hline & Frecuencia & Porcentaje & & Frecuencia & Porcentaje \\
\hline \multirow[t]{2}{*}{ Raglai } & 255 & 0.00 & Mazahua & 8224 & 0.1 \\
\hline & 241 & 0.00 & Triqui & 6545 & 0.1 \\
\hline Mnong & 229 & 0.00 & Huave & 5154 & 0.0 \\
\hline $\mathrm{Ma}$ & 190 & 0.00 & Tojolabal & 4370 & 0.0 \\
\hline Xtieng & 184 & 0.00 & Mayo & 2991 & 0.0 \\
\hline Ta $0 \mathrm{i}$ & 180 & 0.00 & Popoloca & 2717 & 0.0 \\
\hline Co & 125 & 0.00 & Chontal de Oaxaca & 2094 & 0.0 \\
\hline Kho mu & 68 & 0.00 & Pame & 2000 & 0.0 \\
\hline Ngai & 53 & 0.00 & Tepehua & 1685 & 0.0 \\
\hline Lao & 41 & 0.00 & Chontal de Tabasco & 1405 & 0.0 \\
\hline La Chi & 41 & 0.00 & Mame & 1302 & 0.0 \\
\hline Phu La & 28 & 0.00 & Yaqui & 1168 & 0.0 \\
\hline Cho Ro & 26 & 0.00 & Sayulteco & 643 & 0.0 \\
\hline La Hu & 23 & 0.00 & Kanjobal & 589 & 0.0 \\
\hline Cong & 20 & 0.00 & Guariiio & 559 & 0.0 \\
\hline Lo Lo & 19 & 0.00 & Chocho & 463 & 0.0 \\
\hline Chu Ru & 18 & 0.00 & Pima & 356 & 0.0 \\
\hline Lu & 15 & 0.00 & Aguacateco & 156 & 0.0 \\
\hline Si La & 13 & 0.00 & Chichimeca Jonaz & 124 & 0.0 \\
\hline Mang & 9 & 0.00 & Jacalteco & 112 & 0.0 \\
\hline BoY & 9 & 0.00 & Ocuilteco & 104 & 0.0 \\
\hline Khang & 8 & 0.00 & Chontal & 88 & 0.0 \\
\hline Ro Man & 8 & 0.00 & Quiche & 75 & 0.0 \\
\hline Chut & 5 & 0.00 & Kekchi & 64 & 0.0 \\
\hline
\end{tabular}


Lukasz Czarnecki

Cuadro 9. El porcentaje de la población indígena en Vietnam (2009) y México (2010) (continuación)

\begin{tabular}{|c|c|c|c|c|c|}
\hline \multicolumn{3}{|c|}{ Vietnam } & \multicolumn{3}{|c|}{ México } \\
\hline & Frecuencia & Porcentaje & & Frecuencia & Porcentaje \\
\hline Xinh Mum & 4 & 0.00 & Ixcateco & 45 & 0.0 \\
\hline La Ha & 2 & 0.00 & Kumiai & 42 & 0.0 \\
\hline Co Lao & 1 & 0.00 & Texistepequeño & 42 & 0.0 \\
\hline Brau & 1 & 0.00 & Teco & 34 & 0.0 \\
\hline Otros & 325695 & 5.98 & Cucapa & 32 & 0.0 \\
\hline \multirow[t]{14}{*}{ Total } & 5444894 & & Matlatzinca & 30 & 0.0 \\
\hline & & & Chuj & 30 & 0.0 \\
\hline & & & Papago & 26 & 0.0 \\
\hline & & & Kaqchikel & 19 & 0.0 \\
\hline & & & Motocintleco & 13 & 0.0 \\
\hline & & & Seri & 12 & 0.0 \\
\hline & & & Oluteco & 12 & 0.0 \\
\hline & & & Kiliwa & 5 & 0.0 \\
\hline & & & Kikapu & 4 & 0.0 \\
\hline & & & Pai-Pai & 2 & 0.0 \\
\hline & & & |xil & 2 & 0.0 \\
\hline & & & Lacandón & 1 & 0.0 \\
\hline & & & Otros & 16917 & 0.1 \\
\hline & & & $\begin{array}{l}\text { Total población no } \\
\text { hablante español }\end{array}$ & 2374480 & 19.6 \\
\hline
\end{tabular}

Fuente: elaboración propia con datos de IPUMS. Minnesota Population Center. Minneapolis: Universidad de Minnesota, 2015. 


\section{REFLEXIONES FINALES}

La inquietud principal del presente trabajo fue la comparación entre México y la República Socialista de Vietnam en cuanto al impacto del neoliberalismo en la transformación política y sociodemográfica. Al respecto del impacto del neoliberalismo en la transformación política, como resultado se acentuó el debilitamiento del Estado. Las reformas constitucionales en ambos países son llevadas a cabo en función del libre mercado y el capital transnacional. Si bien la primera parte se basó en el análisis de los cambios constitucionales, en la parte posterior se recurrió a las fuentes nacionales e internacionales sobre datos poblacionales. Cabe destacar que las fuentes nacionales difieren de las fuentes internacionales en cuanto a los datos sobre la población en ambos países. Para evitar el sesgo, se recurrió principalmente a los datos nacionales de los censos de 1989, 1999 y 2009 en el caso de Vietnam y los censos de 1990, 2000 y 2010 en el caso de México.

Se encontraron similitudes, pero también diferencias, a pesar del cambio similar en la transformación de la economía cerrada hacia la de libre mercado desde los años ochenta. Primero, se observa la disminución de la población joven y el crecimiento de la población de 60 años y más en ambos países. En Vietnam, el proceso de la disminución de la población de 0-14 es mayor. De ahí, que el índice de envejecimiento de Vietnam sea mayor que el de México.

$\mathrm{El}$ analfabetismo sigue presente sin prácticamente ningún cambio, en el caso de México para el año 2010, el 8.8\% de las personas se registró como analfabetas; mientras que el $4.7 \%$ en Vietnam para el 2009. Respecto a los cambios del porcentaje de las personas y los años de escolarización, se aprecia el crecimiento de las brechas entre las personas que dedican años a la educación. En el caso de México este porcentaje es mayor que en el caso de Vietnam. Por el otro, crece el porcentaje de personas con una educación que supera una década. Además, en los tiempos neoliberales crece la población en la informalidad.

Finalmente, los grupos indígenas sufren exclusión y desigualdad mayores, problemas de salud y carencia educativa de calidad, donde el Estado neoliberal no creó las políticas públicas de igualdad y oportunidad.

El común denominador son los procesos negativos que acompañan las trasformaciones sociodemográficas durante las décadas del neoliberalismo, como son la exclusión social, las desigualdades, la precariedad laboral, profundizando las brechas dentro de los grupos poblacionales, aún más entre la población indígena. Quod erat demonstradum. 
El desafío futuro en la investigación estriba en estudiar y analizar las estrategias de sobrevivencia y adaptación por los grupos vulnerables a las estructuras de exclusión permanente.

\section{BIBLIOGRAFÍA}

Bassols Batalla, Ángel (1981), La República Socialista de Vietnam (con un apéndice sobre la revolución en Laos y la situación en Kampuchea), México, Universidad Nacional Autónoma de México, Instituto de Investigaciones Económicas, pp. 100.

Consejo Nacional de Evaluación de la Política de Desarrollo Social (coneval) (2014), La pobreza en la población indígena de México 2012, México, CONEVAL, pp. 156.

Constitutions of Viet Nam: 1946, 1959, 1980, 1992, 2013 (2015), Hanoi, The Gioi Publishers, pp. 289.

Constitución Politica de los Estados Unidos Mexicanos 1917, México, Cámara de Diputados (consultado el 1 de abril 2016), disponible en <http://www. diputados.gob.mx/LeyesBiblio/htm/1.htm>

Czarnecki, Lukasz (2011), "Olvidados por los olvidados. H'mong en Vietnam y la cuestión mexicana entorno de la metodología etnográfica de Nguyen Tu Chi”, Estudios de Asia y África, vol. XIVI, núm. 144 (1), México, El Colegio de México, pp. 195-221.

D. D. (2005), "Nueva edición del Diario de Debates del Congreso Constituyente de 1916-1917”, en Ignacio Marván, Poder Judicial de la Federación, Introducción y notas, tres volúmenes, México .

De Vos, Susan, Patricio Solís y Verónica Motes de Oca (2004), "Receipt of Assistance and Extended Family Residence Among Elderly Men in Mexico", International Journal Aging and Human Development, vol. 58(1), pp. $1-27$.

General Statistics Office of Vietnam (gso) (2016), Area, Population and Population Density by Province, Hanoi, General Statistics Office of Vietnam (consultado el 10 de abril 2016), disponible en <https://www.gso.gov.vn/ default_en.aspx?tabid=774>

Gomes, Cristina (2007), "Intergenerational Exchanges in Mexico. Types and Intensity of Support", Current Sociology, vol. 55(4), julio, pp. 545-560.

Hanson, Gordon (2010), "Why isn't Mexico Rich", Journal of Economic Literature, vol. 48, núm. 4, December, pp. 987-1004. 
Hong Hoan, Nguyen (2015), "Economic Adjustment and Living Conditions of Young Migrants in Ho Chi Minh City", Vietnam Journal of Family and Gender Studies, vol. 10, núm. 1, pp. 29-56.

Huu Minh, Nguyen y Thi Hong Tran (2015), "Attitudes of Vietnamese Youth toward Marriage and Family Issues", Vietnam Journal of Family and Gender Studies, vol. 10, núm. 1, pp. 15-29.

Instituto Nacional de Estadística y Geografía (INEGI) (2016), México en cifras. Número de personas 2013, México (consultado el 15 de abril de 2016), disponible en <http://www3.inegi.org.mx/sistemas/mexicocifras/default. aspx>

Lincoln, Martha (2008), "Report from the Field: Street Vendors and the Informal Sector in Hanoi", Dialectical Anthropology, vol. 32, núm. 3, pp. 261-265.

Long, Tran Quy (2013), "Structural Changes within Vietnamese Household and their Correlations with Demographic and Social Elements", Vietnam Journal of Family and Gender Studies, vol. 8, núm. 2, pp. 24-39.

My Hanh, Nguyen Thi (2016), "Investiture Beseeching in Vietnam-China Diplomatic Relationship in Feudal Age", Asian Culture and History, vol. 8, núm. 1, pp. 17-27.

Naciones Unidas (2013), World Population Ageing, New York, Department of Economic and Social Affairs, Population Division, UN.

Navarrete, Emma Liliana (2012), "Jóvenes universitarios mexicanos ante el trabajo", Revista Latinoamericana de Población, vol. 6, núm. 10, enerojunio, pp. 119-140.

Navarrete, J. Manuel y Fernando Aragón-Durand (2011), "Garment Maquiladoras in the South: A Closer Look at the Mayan Maquiladora Workforce”, Latin American Perspectives, vol. 38, núm. 5, pp. 93-109.

Nguyen Anh, Dang (2014), "Social Protection in Vietnam: Issues, Challenges and Prospects", Vietnam Journal of Family and Gender Studies, Institute for Family and Gender Studies, Vietnam Academy of Social Science, vol. 9, pp. 3-15.

Nguyen Dac, Simone, A. y Mariela Álvarez (1991), “Analogías y diferencias en el desarrollo agrícola de dos regiones tropicales: Los Altos de Chiapas (México) y Nghe-Tinh (Trung-Bo, Vietnam)", Estudios de Asia y África, vol. 26, núm. 1 (84), pp. 112-138.

Profiles of Ageing (2015), disponible en https://esa.un.org/unpd/popdev/Profilesofageing2015/index.html 
Smith, Clint E. (2000), Inevitable Partnership. Understanding Mexico-U.S. Relations, London, Lynne Rienner Publushers.

Solís, Patricio (2015), "Desigualdad vertical y horizontal en las transiciones educativas en México", en Roberto Vélez Grajales, Juan Enrique Huerta Wong y Raymundo M. Campos Vázquez (eds.), México, ¿el motor inmóvil?, México, Centro de Estudios Espinosa Yglesias, A.C., pp. 47-95.

Turner, Sara y Jean Michaud (2008), "Imaginative and Adaptive Economic Strategies for Hmong Livelihoods in Lào Cai Province, Northern Vietnam”, Journal of Vietnamese Studies, vol. 3, núm. 3, pp. 158-90.

Van de Walle, Dominique y D. Gunewardena (2011), "Sources of Ethnic Inequality in Viet Nam", Journal of Development Economics, vol. 65, núm. 1, pp. 177-207.

Xuan Nam, Pham (2001), Rural Development in Vietnam, Hanoi, Social Science Publishing House, pp. 248. 